3. Ueber eine Erscheinung an curarisirten Fröschen.

Wohl Jeder, welcher häufig Frösche zu curarisiren Gelegenheit hat, wird, wenn die Temperatur nicht zu niedrig war, bemerkt haben, dass wenn man den Frosch nach eingetretener Lähmung tödtet, das Blat eine sehr livide Farbe zeigt, ganz wie bei dureh Curare erstickten Warmblïtern. An der Luft röthet sich das Blut schnell. Meines Wissens hat noch Niemand auf diese Erscheinung aufmerksam gemacht, und doch ist sie nicht ganz ohne Bedeutung. Sie lehrt nämlich, dass der Kaltblüter nach Unterbrechung seiner Athmung, den in seinem Blute vorräthigen Sauerstoff nahezu ebenso schnell verbraucht wie der Warmblüter, dass also die relative Unabhängigkeit des Kaltblüters von der Respiration nicht etwa darauf beruht, dass er an seinem Sauerstoff länger zehrt, sondern darauf, dass seine Spaltungsprocesse, auf denen, wie ich 1867 zuerst für die Muskeln, Liebig und Pfliuger später allgemeiner gezeigt haben, das Leben beruht, langsamer verlaufen, während der zum Aufbau der spaltbaren Substanzen erforderliche Sauerstoff stets aus dem Blute sofort verzehrt wird. Das Blut ist also für Sauerstoff uiberhaupt kein Vorrathsmagazin, sondern nur ein Transportmittel.

(Aus dem physiologischen Laboratorium in Zürich.)

\title{
Ueber Secretionsströme an der Zunge des Frosches, nebst Bemerkungen über einige andre Secretionsströme.
}

Von

\section{Termann und B. Luchsinger.}

Die Zunge des Frosches schien uns wegen ihres Drüsenreichthums, und wegen der Leichtigkeit, mit der ihre Nerven präparirt und isolirt gereizt werden können ${ }^{1}$ ), ein sehr geeignetes Object um

1) Vgl. Lépine, über Entstehung und Verbreitung des thierischen Zuckerfermentes. Vorgelegt von Lu d wig. Ber. d. sächs. Ges. d. Wiss. 1870. p. 322. 
weitere Studien über secretorische Ströme anzustellen (vgl. dies Archiv, Band XVII, p. 291, 310). Diese Erwartung wurde in der That erfillt.

Von den Nerven der Zunge richteten wir aus naheliegenden Gründen vor allem unsere Aufmerksamkeit auf den R. glossopharyngeus n. vagi, der beim Frosche die Rollen des Lingtualis und Glossopharyngeus der Säugathiere in sich vereinigt. Ueber die Präparation dieses Nerven brauchen wir keine Bemerkungen zu machen. Später wurde auch der Hypoglossus in das Bereich der Untersuchung gezogen. Den Thieren, mit Ausnahme einer Temporaria stets Esculenten von ziemlicher Grösse, wurde vor dem Versuche Hirn- und Rückenmark zerstört, in manchen Fällen auch vorher Curare gegeben (bei den Hypoglossusversuchen natürlich jedesmal); die Versuche belehrten uns, dass wenigstens in den mässigen von uns angewandten Dosen das Curare ohne Einfluss auf das Resultat ist.

Das Thier wurde mit dem Riicken auf eine Porzellanplatte gelegt, die Zunge mit Schonung vorgezogen und auf eine Glasplatte gebreitet, welche zwei aufeinandergekittete parallele Korkleistchen zur Fixirung der beiden Zungenspitzen mittels Stecknadeln, oder durch blosse Adhäsion, besass. In den meisten Fällen wurde iibrigens die ganze hintere Hälfte des Rumpfes sammt den Hinterbeinen entfernt. Die ableitenden Electroden, gewöhnliche Thonspitzen, wurden den beiden Seitenrändern der Zunge, meist in der Mitte ihrer Länge, symmetrisch angelegt. Als Reizelectroden für die feinen Nerven benutzten wir feine Stecknadeln, welche an die Spitzen der (horizontalen) Zinkdrähte des in diesem Archiv Bd. VII p. 332 beschriebenen und abgebildeten Electrodenstativs so angelöthet waren, dass sie vertical herabragten; die unteren Enden dieser Nadeln waren hakenförmig ein wenig aufgebogen, um den Nerven zu tragen. Die beiden Electrodennadeln hatten einen gegenseitigen Abstand von $2-3 \mathrm{~mm}$. Jeder der beiden Nerven (der rechte und der linke) war auf ein solches Electroden-Nadelpaar aufgelegt, und beide Electrodenpaare mittels einer Wippe ohne Kreuz mit dem Schliussel zum Tetanisiren verbunden. Sowohl um die Vertrocknung zu verhuten, als um der Gefahr unipolarer Wirkungen besser zu entgehen, verdickten wir die zarten, iibrigens recht ausdauernden Nerven mit einem angelegten Stiuck eines anderen dünnen Froschnerven (Ischiadicusäste). Dass wir 
durch die gewöhnlichen Vorsichtsmassregeln und Controllen uns vor Täuschungen durch Stromesschleifen, unipolare Wirkungen u. dgl. sorgfältig schützten, bedarf wohl kaum der Erwähnung; übrigens sind diese Gefahren hier gering, wir hielten uns meist im Gebiete der schwachen Reizströme, und ein grosser Theil der beobachteten Vorgänge, deren Intensität sehr beträchtlich ist, spielt sich ibberhaupt erst nach Aufhören der Reizströme ab, oder ist wenigstens von der Fortdaner derselben unabbängig.

Zunächst einige Bemerkungen über den Ruhestrom der Zungenschleimhant. Da ein Abpräpariren derselben nicht wohl möglich ist, so beschränkten wir uns darauf, von der Oberfläche der unversehrten Zunge einerseits, und von irgend einem enthäuteten und sonst unversehrten Körpertheil des Frosches (z. B. Rückenmuseulatur) andererseits abzuleiten. Erstere verhält sich dabei regelmäsig kräftig negativ gegen letztere; die Ablenkung beträgt mehrere Hundert (bis 700) Scalentheile, die Kraft beträgt etwa 0,01-0,02 Dan. Die Zungenschleimhant ist also, wie die äussere Haut und wie nach Ros enthal die Magen- und Darmschleimhaut, Sitz einer von aussen nach innen gerichteten („einsteigenden") electromotorischen Kraft. Leitet man von der Zunge und der äusseren Haut am unversehrten Thiere ab, so erhält sich erstere mässig positiv, d. h. die Kraft des Hautstroms ist grösser als die der Zungensehleimhaut. Leitet man ferner von einer unversehrten und einer mit Kreosot geätzten Stelle der Zunge ab, so verhält sich erstere schwach negativ oder neutral, ein Zeichen, dass die Aetzung die Musculatur mit ergreift, denn sonst müsste der volle Strom der Schleimhaut auftreten; der volle Muskelstrom aber kommt in diesem Falle nicht zur Wirkung, denn die Schleimbant verhält sich ziemlich stark positiv gegen einen künstlichen Querschnitt der Zunge, d. h. die Kraft des Muskelstroms ist grösser als die des Schleimhautstroms.

Bei den Reizversuchen fällt der Ruhestrom der Schleimhaut selbstverständlich ausser Betracht, da von zwei symmetrischen Schleimbautpuncten abgeleitet ist. Natürlich .ist trotzdem, wegen der Ungleichheit der Kraft .an verschiedenen Schleimhautstellen stets ein schwacher Differenzstrom vorhanden, den wir in den Versuchsbeispielen der Vollständigkeit halber angegeben haben (,Ruhestrom"), obgleich er keine Rolle spielt.

Es bedarf kaum der Erwähnung, dass wenn bei Reizung des rechten Nerven eine Ablenkung nach links auftritt, dies einen von 
aussen nach innen gerichteten Strom der gereizten Schleimhautseite anzeigt u. s. w. Um schleppende Benennungen zu vermeiden, wollen

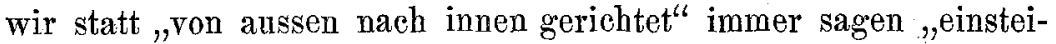
gend", und im entgegengesetzten Fall ,aussteigend". Ferner haben wir in den Beispielen besserer Uebersicht halber, statt die Richtung des Stroms nach rechts oder links, wie in unserer früheren Arbeit, durch Pfeile zu bezeichnen, gleich die einsteigende oder aussteigende Richtung mit Bezug auf die gereizte Schleimhaut angegeben, und zwar haben wir die einsteigende als die positive Richtung bezeichnet, weil sie mit der des Ruhestroms der Schleimhaut übereinstimmt'), die aussteigende als die negative.

Das völlig regelmässige Resultat der Reizversuche lässt sich nun folgendermassen ausdrücken:

Die Reizung eines Glossopharyngeus bewirkt in der erregten Schleimhaut nach einem dentlichen Latenzstadium einen zuerst einsteigenden Strom, der aber sofort einem aussteigenden Platz macht; dann aber stellt sich, gleichgültig ob die Reizung schon beendet ist oder fortgesetzt wird, wieder ein mächtiger einsteigender Strom ein, der die Reizung, falls sie nicht fortgesetzt wird, lange überdauert, langsam ein Maximum erreicht, und dann äusserst langsam wieder schwindet.

Um diese Erscheinungen zu veranschaulichen, führen wir zunächst einige Beispiele aus einer grösseren Zahl von Versuchen an.

1. Beispiel (28. Mai). Grosse Esculenta, kein Curare, Glossopharyngei. (Ruhestrom $\rightarrow 196$ sc.)

\begin{tabular}{|c|c|c|c|c|c|}
\hline \multirow[b]{2}{*}{$\begin{array}{l}\text { Rollen- } \\
\text { abstand. }\end{array}$} & \multirow[b]{2}{*}{$\begin{array}{c}\text { Gereizter } \\
\text { Nerv }\end{array}$} & \multicolumn{3}{|c|}{ Ablenkung durch die Reizung } & \multirow[b]{2}{*}{$\begin{array}{c}\text { Kraft der 3. Phase } \\
\text { in Daniells }\end{array}$} \\
\hline & & $\begin{array}{l}\text { 1. Phase } \\
\text { sc. }\end{array}$ & $\begin{array}{l}\text { 2. Phase } \\
\text { sc. }\end{array}$ & $\begin{array}{c}\text { 3. Phase } \\
\text { sc. }\end{array}$ & \\
\hline $\begin{array}{l}"{ }_{0} \\
" 0_{1} \\
" 0_{2}\end{array}$ & $\begin{array}{l}\text { links } \\
\text { rechts } \\
\text { links } \\
\text { rechts } \\
\text { links } \\
\text { rechts } \\
\text { links } \\
\text { rechts } \\
\text { links } \\
\text { rechts }\end{array}$ & $\begin{array}{l}+2 \\
+15 \\
+8 \\
+15 \\
+15 \\
+52 \\
+3 \\
+42 \\
+1 \\
+35\end{array}$ & $\begin{array}{c}-148 \\
-\quad 73 \\
-152 \\
-\quad 67 \\
-109 \\
\text { nicht be } \\
-\quad 82 \\
-\quad 57 \\
-\quad 5\end{array}$ & $\begin{array}{c}+289 \\
+334 \\
+199 \\
+172 . \\
+268 \\
\text { bachtet } \\
+30 \\
+\end{array}$ & $\begin{array}{c}0,0037 \\
0,0044 \\
0,0024 \\
0,0019 \\
0,0035 \\
- \\
- \\
0,0003 \\
- \\
-\end{array}$ \\
\hline
\end{tabular}

1) Dass diese Bezeichnung nichts zu thum hat mit der Annahme einer positiven oder negativen Schwankung des Ruhestromes im Sinne der Präexistenzlehre, hat der Eine von uns schon frither hervorgehoben, dies Archiy Bd. XVII, p. 301. 


\begin{tabular}{|c|c|c|c|c|c|}
\hline \multirow{2}{*}{$\begin{array}{l}\text { Rollen- } \\
\text { abstand }\end{array}$} & \multirow[b]{2}{*}{$\begin{array}{c}\text { Gereizter } \\
\text { Nerv }\end{array}$} & \multicolumn{3}{|c|}{ Ablenkung durch die Reizung } & \multirow[b]{2}{*}{$\begin{array}{c}\text { Kraft der 3. Phase } \\
\text { in Daniells }\end{array}$} \\
\hline & & $\begin{array}{l}\text { 1. Phase } \\
\text { sc. }\end{array}$ & $\begin{array}{l}\text { 2. Phase } \\
\text { sc. }\end{array}$ & $\begin{array}{c}\text { 3. Phase } \\
\text { sc. }\end{array}$ & \\
\hline
\end{tabular}

Ableitungsstellen etwas näher der Zungenwurzel, Reizstelle an den

Nerven etwas nach der Pexipherie verschoben (Ruhestrom $\rightarrow 14$ sc.).

\begin{tabular}{|c|c|c|c|c|}
\hline $\begin{array}{l}100 \\
" \\
"\end{array}$ & $\begin{array}{l}\text { links } \\
\text { rechts } \\
\text { links } \\
\text { rechts }\end{array}$ & $\begin{array}{c}+87 \\
\text { nicht bec } \\
+94 \\
+63\end{array}$ & $\begin{array}{r}-185 \\
\text { obachtet } \\
-\quad 65 \\
-\quad 37\end{array}$ & $\begin{array}{l}+311 \\
+398 \\
+313 \\
+460\end{array}$ \\
\hline
\end{tabular}

Beide Nerven unterhalb der Reizstelle zerquetscht.

\begin{tabular}{c|l|l|ll||l|l|l}
100 & links & & 0 & 0 & 0 & 0 \\
$m$ & rechts & 0 & 0 & 0 & 0 \\
\hline
\end{tabular}

2. Beispiel (31. Mai). Esculenta, der gleiche Versuch, kein Curare.

a) Ableitung vom mittleren Theil (Ruhestrom $\leftarrow 113 \mathrm{sc}$.).

\begin{tabular}{l|l|l||c||c|c}
140 & links & $+*$ & -21 & +102 & 0,0017 \\
100 & rechts & +1 & -73 & +167 & 0,0032 \\
$"$ & links & $+1 / 2$ & -77 & +75 & 0,0018 \\
& rechts & $+1 / 2$ & -63 & +88 & 0,0028
\end{tabular}

b) Ableitung vom gespaltenen Theil.

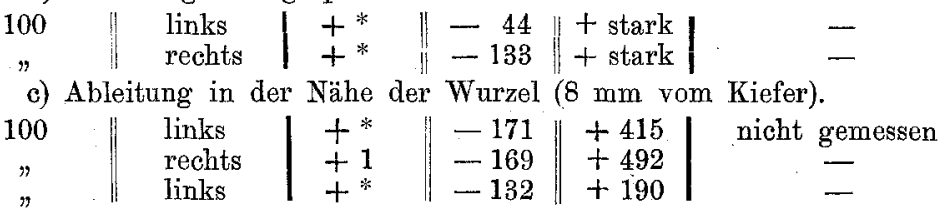

3. Beispiel (5. Juni). Esculenta. Der gleiche Versuch.

a) Ableitung vom mittleren Theil (Ruhestrom $\leftarrow 16$ ).

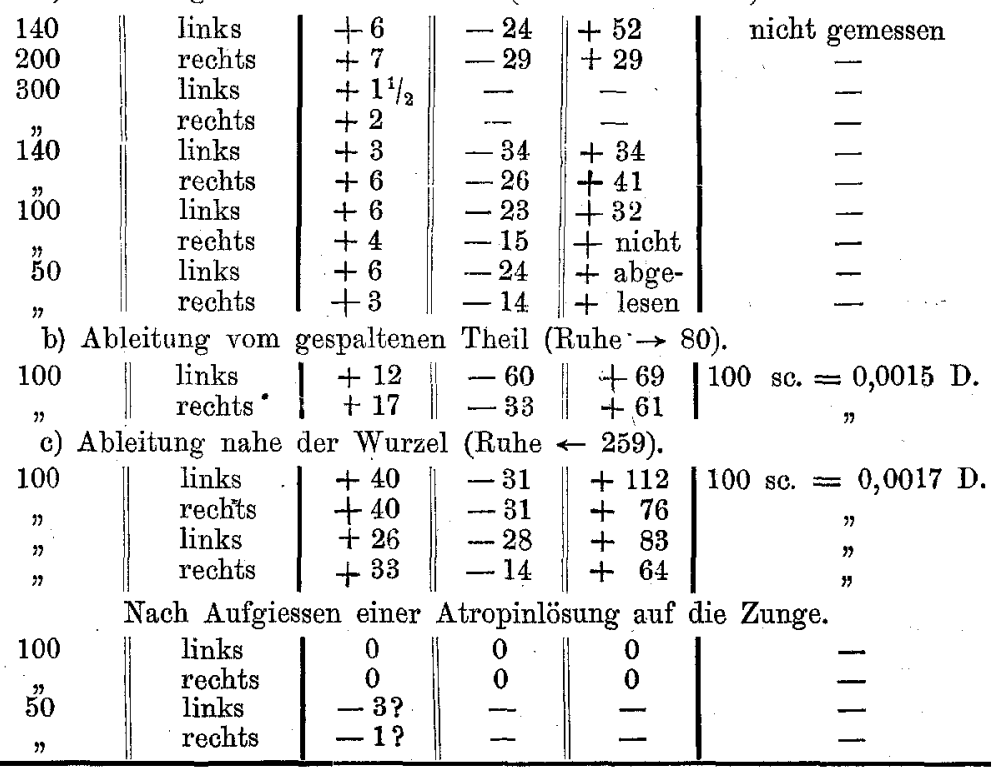




\begin{tabular}{|c|c|c|c|c|c|}
\hline \multirow[b]{2}{*}{$\begin{array}{l}\text { Rollen- } \\
\text { abstand }\end{array}$} & \multirow[b]{2}{*}{$\begin{array}{c}\text { Gereizter } \\
\text { Nerv }\end{array}$} & \multicolumn{3}{|c|}{ |Ablenkung durch die Reizung } & \multirow[b]{2}{*}{$\begin{array}{l}\text { Kraft der 3. Phase } \\
\text { in Daniells }\end{array}$} \\
\hline & & $\begin{array}{l}\text { 1. Phase } \\
\text { sc. }\end{array}$ & $\begin{array}{l}\text { 2. Phase } \\
\text { sc. }\end{array}$ & $\begin{array}{c}\text { 3. Phase } \\
\text { sc. }\end{array}$ & \\
\hline
\end{tabular}

4. Beispiel (9. October). Esculenta.

\begin{tabular}{|c|c|c|c|c|c|}
\hline $\begin{array}{c}140 \text { moment. } \\
" \text { contin. } \\
" \text { " } " \\
100 \text { moment. } \\
" \text { contin. } \\
", \quad "\end{array}$ & $\begin{array}{l}\text { rechts } \\
\text { links } \\
\text { rechts } \\
\text { links } \\
\text { rechts } \\
\text { links } \\
\text { rechts } \\
\text { links } \\
\text { links }\end{array}$ & $\begin{array}{l}+53 \\
+\quad 12 \\
+\quad 77 \\
+\quad 30 \\
+\quad 59 \\
+\quad 76 \\
+\quad 87 \\
+103 \\
+\quad 15\end{array}$ & $\begin{array}{l}-0 \\
-13 \\
-12 \\
-51 \\
+\quad 2 \\
-19 \\
+26 \\
-12 \\
-11\end{array}$ & $\begin{array}{l}+61 \\
+\quad 37 \\
+131 \\
+160 \\
+\quad 22 \\
+\quad 91 \\
+131 \\
+254 \\
+\quad 22\end{array}$ & $\begin{array}{c}100 \text { sc. }=0,0017 \mathrm{D} . \\
" \\
" \\
" \\
" \\
" \\
"\end{array}$ \\
\hline
\end{tabular}

5. Beispiel (30. Juli). Esculenta, curarisirt.

\begin{tabular}{|c|c|c|c|c|c|}
\hline $\begin{array}{c}140 \\
100 \\
"\end{array}$ & $\begin{array}{l}\text { links } \\
\text { rechts } \\
\text { links } \\
\text { rechts }\end{array}$ & $\begin{array}{l}+10 \\
+35 \\
+23 \\
+40\end{array}$ & $\begin{array}{r}-88 \\
-190 \\
-\quad 80 \\
-238\end{array}$ & $\begin{array}{l}+175 \\
+310 \\
+270 \\
+455\end{array}$ & $\begin{array}{c}100 \text { sc. }=0,0044 \mathrm{D} . \\
" \\
" \\
"\end{array}$ \\
\hline \multicolumn{6}{|c|}{ Nach Aufgiessen von Atropin. } \\
\hline 100 & links & +1 & -41 & +36 & - \\
\hline$n$ & rechts & +1 & -60 & +139 & - \\
\hline \multicolumn{6}{|c|}{ Mehr Atropin. } \\
\hline 100 & links & +1 & -12 & +86 & - \\
\hline & rechts & $+*$ & -40 & +46 & - \\
\hline 50 & links & +1 & -3 & +44 & 一 \\
\hline " & rechts & $+*$ & -22 & +14 & - \\
\hline
\end{tabular}

6. Beispiel (31. Juli). Esculenta, vor dem Versuch atropinisirt und curarisirt.

\begin{tabular}{l|l|l||c||c|c}
140 & links & 0 & 0 & 0 & 100 sc. $=0,0022 \mathrm{D}$. \\
100 & rechts & 0 & 0 & 0 & $"$ \\
$"$ & links & 0 & -18 & 0 & $"$ \\
$"$ & rechts & 0 & $-?$ & 0 & $"$ \\
$"$ & links & 0 & -20 & - & $"$ \\
50 & rechts & 0 & $-?$ & - & $"$ \\
$"$ & links & +3 & $-3 ?$ & $-?$ & $"$
\end{tabular}

7. Beispiel (5. August). Esculenta, vor dem Versuch atropinisirt und curarisirt.

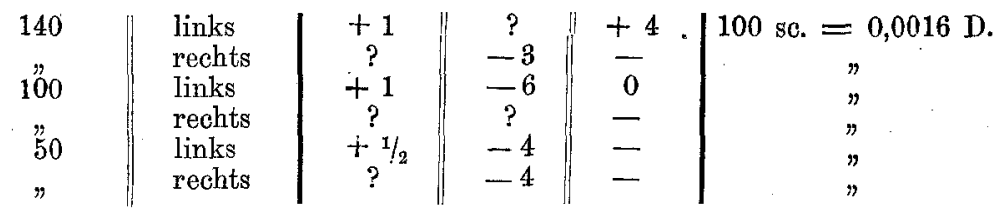

8. Beispiel (16. August). Esculenta, curarisirt; an dem Thiere ist unmittelbar vorher ein Hypoglossusversuch gemacht worden (hier folgen nur die Resultate der Glossopharyngensreizung).

\begin{tabular}{c||l|c||c||c|c}
100 & links & +160 & +54 & +320 & 100 sc. $=0,0019 \mathrm{D}$ \\
& rechts & +59 & +22 & +210 & $"$ \\
50 & links & +46 & +5 & +196 & $"$ \\
$"$ & rechts & +70 & +40 & +200 & $"$
\end{tabular}


Die Beispiele sind so ausgewählt, dass sie für die nun folgenden specielleren Bemerkungen Belege darbieten.

Wegen des relativ geringen Widerstandes der feuchten Zunge entsprechen den ziemlich bedeutenden Ablenkungen doch nur geringe electromotorische Kräfte; in einigen Versuchen (Beispiel 1 und 2) haben wir dieselben, und zwar aus naheliegenden Gründen nur für die andauernde dritte Phase, direct gemessen, indem wir nach jedem Versuch compensirten und immer die Differenzen nahmen. In den meisten Fällen begnïgten wir uns damit, nur Eine Verhältnisszahl zwischen Ablenkung und electromotorischer Kraft festzustellen, aus welcher der Leser die electromotorischen Kräfte sich ausrechnen kann. Die grösste iiberhaupt beobachtete Kraft der dritten Phase findet sich beim rechten Glossopharyngeus im Beispiel 5; bei mässiger Reizung (100 mm Rollenabstand) betrug hier die Kraft $0,0200=1 / 50$ Daniell.

Das Grössenverhältniss der drei successiven Ablenkungen ist wie man sieht, sehr wechselnd. Die erste, welcher stets ein dentliches Latenzstadium vorangeht, schwankt von 0 bis gegen 100 sc., ja einmal (Beispiel 8) 160 sc. In den Fällen, in denen es die Grösse Null hat, ist es trotzdem sehr deutlich markirt; man sieht nämlich die folgende, aussteigende Ablenkung ganz entschieden verzögert und gleichsam im Kampf begriffen mit einer einsteigenden. Mit vollem Recht durften wir daher solche Fälle so bezeichnen, dass wir die Phase durch ein Pluszeichen andeuteten, aber statt der Zahl ein * beifügten, als Zeichen, dass die Ablenkung nur durch Verzögerung der entgegengesetzten sich zu erkennen gab (Beispiel 2 und 5). Aehnliche Grössenschwankungen zeigt auch die zweite Phase. Während sie in manchen Fällen eine absolute Grösse von fast 250 sc. erreicht, markirt sie sich in Beispiel 4 und 8 nur durch einen Rückgang der einsteigenden 'Ablenkung. gegen die Null, ohne dass aber letztere erreicht oder gar uiberschritten wurde, so dass ihr absoluter Betrag gleichsinnig bleibt mit der ersten Phase. (Natürlich bezeichnen alle Zahlen die $\mathrm{Ab}$ lenkung von der Ruhestellung vor der Reizung aus gerechnet.)

Die dritte Phase entwickelt sich stets im Vergleich mit den beiden ersten relativ langsam; ihre Grösse und ihr zeitlicher Verlauf sind fast ganz unabhängig davon, ob man die Reizung schon während der zweiten Phase beendet, oder sie noch. während der 
dritten mehr oder weniger lange fortsetzt. Es gewährt einen sehr seltsamen Anblick, wenn man, wie wir es zur Schonung des Nerven meistens thaten, schon in der zweiten Phase die Reizung beendigt, nun noch eine neve mächtige Bewegung von zuweilen $400-500$ sc. uiber den Nullpunct hinaus langsam sich einstellen zu sehen.

Wiederholt man die Reizung des gleichen Nerven noch ehe die dritte Phase wieder verschwunden ist (Beispiel 4, am Schluss), so stellen sich wiederum alle drei Phasen, aber sehwächer ein, und die grösste relative Schwächung zeigt die dritte. Auch wenn man die Herstellung des ursprtinglichen Zustandes völlig abwartet, ist die neue Reizung von geringerem Erfolge, jetzt aber trifft die Abnahme alle drei Phasen ziemlich gleichmässig.

Reizt man iiberhaupt nur einen kurzen Moment, so dass die Reizung: schon vor Ende des Latenzstadiums beendet ist, so sieht man trotzdem nach Schluss der Reizung alle drei Phasen sich abspielen, aber schwächer als bei anhaltender Reizung, und namentlich ist die dritte Phase relativ schwach entwickelt (Beispiel 4).

Wer diese Versuche anstellt, wird sofort den Eindruck empfangen, dass die dritte Phase nichts anderes ist, als die Fortsetzung der ersten, und dass nur eine vorübergehende Uebercompensation durch einen zweiten, entgegengesetzt gerichteten Strom sich als zweite Phase einschiebt. Besonders deutlich wird dies durch die langsame Entwicklung der dritten Phase im Vergleich mit den beiden andern; diese Entwicklung ist eben identisch mit dem Schwinden einer Gegenwirkung, und alle Wirkungen dieser Art schwinden viel langsamer als sie entstehen.

Das Gesetz unsrer Erscheinung lässt sich also auch folgendermassen ausdrücken:

Tetanisiren des Glossopharyngeus bewirkt an der Froschzunge einen starken, die Reizung sehr lange tiberdawernden, und sehr langsam schwindenden, einsteigenden Strom. Bald nach seinem Beginn tritt jedoch noch ein zweiter, bald voribergehender, aussteigender, schneller entstehender als schwindender Strom auf, welcher den ersteren fast stets voriibergehend iubercompensirt, aber zuweilen nur eine vorübergehende Schwächung desselben bewirkt.

Man kann begreiflicherweise nicht wissen, auf welche Zeit das wahre Maximum des ersten Stromes fällt, sein scheinbares Maximum, welches sehr spät eintritt, sagt hierïber durchaus Nichts aus. 
Wiederum haben wir also hier ein ähnliches Factum, wie es der Eine von uns an der äusseren Haut des Frosches fand: zwei einander entgegengesetzte Ströme von verschiedenem zeitlichen Verlauf. Wiederum ist der dominirende Strom wie dort einsteigend gerichtet, aber der Gegenstrom, der dort an manchen Stellen gânz fehlte, und wo vorhanden dem Hauptstrom stets voranging, beginnt hier etwas später als dieser, weicht ihm aber freilich bald auch hier.

Es entsteht nun vor Allem die Frage, ob wir es auch hier mit Secretionsströmen zu thun haben. Eine reichliche Secretion einer sehr stark alkalischen schleimigen Flüssigkeit tritt allerdings bei der Reizung auf, wie schon Lépine beobachtet hat. Ob aber die Secretion mit dem Strome direct zusammenhängt, lässt sich hier, wie an der Haut, nicht ohne Weiteres feststellen. Wir versuchten die Wirkung des Atropins, und es zeigte sich sofort, dass dasselbe fast stets den eben noch kräftigen Strom beträchtlich schwächt oder ganz zum Verschwinden bringt (Beispiel 3 und 5); wird das Thièr vor dem Versuch atropinisirt, so ist der Strom von vorn herein schwach oder fehlend (Beispiel 6 und 7), ein Beweis, dass jene Schwächung nicht von Zeitverlust oder Ermiidung herriihte. Curare ist in mässigen Dosen ohne Einfluss. Diese Thatsachen sprechen sehr dafür, dass es sich um secretorische Ströme handelt.

Die Wirkung des Atropins auf die beiden entgegengesetzten Ströme ist, wie man aus den Beispielen ersieht, insofern etwas verschieden, als der zweite Strom etwas weniger geschädigt wird, als der Hauptstrom. Indess wäre der Schluss, dass ersterer einem andern Organ angehört als der letztere, doch selbst dann nicht gerechtfertigt, wenn der genannte Unterschied prägnanter wäre. Es bleibt somit auch hier, wie bei der Haut, die Frage offen, ob beide Ströme vielleicht versehiedenen Drüsengattungen oder iiberhaupt nur der eine den Drüsen angehört, oder ob beide durch verschiedene Nervenfasern der gleichen Drüse hervorgebracht werden (wobei man an secretorische und Hemmungsnerven, oder an secretorische und Gefässnerven, oder auch an secretorische und trophische Fasern, im Sinne der nenesten Untersuchungen Heidenhai n's, denkën kann). Ueberhaupt ist die Zeit für eine theoretische Deutung der Secretionsströme noch nicht gekommen.

In Bezug auf das Verhältniss dieser Ströme zu den von uns 
beschriebenen an der Haut der Warmblïter erinnern wir daran, dass wir an diesen nichts yon Doppelsinnigkeit beobachtet haben; der ganze Verlauf dieser letzteren Ströme ist rascher, das Latenzstadium sehr kurz (ausser bei Atropinisirung; vgl. a. a. 0.), und ein Kampf zweier Ströme würde uns, obgleich die Ablenkungen wegen des grossen Widerstands ziemlich klein warẹn, kaum haben entgehen können.

Wir habẻn auch einige Versuche an der Zunge des Hundes und der Katze angestellt. Die Thiere waren curarisirt und unter künstlicher Respiration. Die Zunge wurde aus dem weit geöffneten Maul hervorgezogen, und mittels eines durch die Spitze gezogenen Fadens und eines Stativs befestigt. Die ableitenden Electroden wurden symmetrisch dem Zungenrücken in der Nähe des Randes angelegt. Reizung des Lingualis ergab keine dentlichen Resultate, dagegen sahen wir bei einem Hunde auf Reizung des Hypoglossus völlig regelmässig einen von aussen nach innen gerichteten Strom auftreten, der, nacbdem er eine Ablenkung von 10-25 sc. hervorgebracht, stockte und dann in gleichem Sinne langsam noch bedeutend anwuchs und die Reizung lange uiberdanerte; auch hier also schien ein Gegenstrom durch das kurze Stehenbleiben angedeutet. Wir theilen dieses vorläufig vereinzelte Resultat nur aus einem sogleich anzugebenden äusseren Grunde schon jetzt mit.

Die genannte Wirksamkeit des Hypoglossus veranlasste uns, anch am Hypoglossus des Frosches Versuche anzustellen. Dieselben ergaben, dass der Hypoglossus genau die gleiche Wirkung hat, wie der Glossopharyngeus, jedoch meist viel schwächer als letzterer. Indess ist es uns bei einem Frosche begegnet, dass wir, aber nur auf der einen Seite, Glossopharyngeus und Hypoglossus in gleichem Grade wirksam fanden. Diejenigen Fasern also, welche die von uns beschriebenen Ströme hervorbringen, sind auf beide Nerven in ungleichem, aber individuell (sogar unsymmetrisch) wechselndem Maassstabe vertheilt. Wir führen ein Beispiel an, in welchem zuerst die Hypoglossi, dann die Glossopharyngei gereizt wurden (der zweite Theil dieses Versuchs figurirt schon oben als Beispiel 7). 
9. Beïspiel (16. August). Esculenta, curarisirt (Ruhestrom $\rightarrow 423$ sc.).

\begin{tabular}{|c|c|c|c|c|c|}
\hline \multirow[b]{2}{*}{$\begin{array}{l}\text { Rollen- } \\
\text { abstand }\end{array}$} & \multirow[b]{2}{*}{$\begin{array}{c}\text { Gereizter } \\
\text { Nerv }\end{array}$} & \multicolumn{3}{|c|}{ Ablenkung durch die Reizung } & \multirow[b]{2}{*}{$\begin{array}{c}\text { Kraft der 3. Phase } \\
\text { Dan. }\end{array}$} \\
\hline & & $\begin{array}{c}\text { 1. Phase } \\
\text { se. }\end{array}$ & {$\left[\begin{array}{c}\text { 2. Phase } \\
\text { sc. }\end{array}\right.$} & $\begin{array}{c}\text { 3. Phase } \\
\text { sc. }\end{array}$ & \\
\hline $\begin{array}{c}140 \\
100 \\
50_{1} \\
" \\
" \\
50_{2} \\
"\end{array}$ & \begin{tabular}{|l} 
Hypoglossus \\
lipks \\
rechts \\
links \\
rechts \\
links \\
rechts \\
links \\
rechts \\
links \\
rechts \\
\end{tabular} & $\begin{array}{l}- \\
+1 \\
+1 \\
+7 \\
+1 \\
+3 \\
+2 \\
+6 \\
+2 \\
\end{array}$ & $\begin{array}{l}- \\
-8 \\
-\quad 8 \\
-\quad 7 \\
=1 \\
-18 \\
=3 \\
-15 \\
-\quad 4 \\
\end{array}$ & 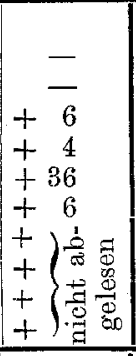 & $\begin{array}{c}100 \text { sc. }=0,0019 \mathrm{D} . \\
" \\
" \\
" \\
" \\
" \\
" \\
" \\
" \\
"\end{array}$ \\
\hline $\begin{array}{c}100 \\
50 \\
"\end{array}$ & $\begin{array}{l}\text { Glossophar. } \\
\text { links } \\
\text { rechts } \\
\text { links } \\
\text { rechts }\end{array}$ & $\begin{array}{r}+160 \\
+\quad 59 \\
+\quad 46 \\
+\quad 70\end{array}$ & $\begin{array}{l}-54 \\
-22 \\
-\quad 5 \\
-40\end{array}$ & $\begin{array}{l}+320 \\
+214 \\
+196 \\
+200\end{array}$ & $\begin{array}{l}" \\
" \\
"\end{array}$ \\
\hline
\end{tabular}

Unsere gemeinsamen Untersuchungen können zunächst nicht fortgesetzt werden, da der Eine von uns eine Professur in Bern iibernimmt. Wir theilen deshalb anhangsweise noch einige fragmentarische Thatsachen mit, welche sich an unsere Arbeit iiber die Secretionsströme der Haut anschliessen; wäre das genannte Hinderniss nicht vorhanden, so hätten wir mit dieser Mittheilung gewartet bis uns ein reicheres Material vorlag.

Wir haben uns anfang's auf die Fussballen der Katze beschränkt, weil hier mit Sicherheit auf sichtbare Secretion gerechnet werden kann, und weil wir einen innigen Zusammenhang: der Ströme mit den Drüsen vermutheten. Einige seitdem angeśtellte Versuche bestätigen nun in der That, dass die Entwicklung der Ströme mit der der Drüsen ungefähr gleichen Schritt hält. Während bei Katzen der Strom mit gleicher Regelmässigkeit entwickelt ist wie die Secretion, fehlte er vollständig an einem ganz jungen (4tägigen), nicht schwitzenden Hündehen und an einer Ratte; an einer andern (albinotischen) Ratte dagegen war er, wenn auch schwach, so doch vollkommen regelmässig vorhanden. Wir theilen letzteren Versuch noch mit. 


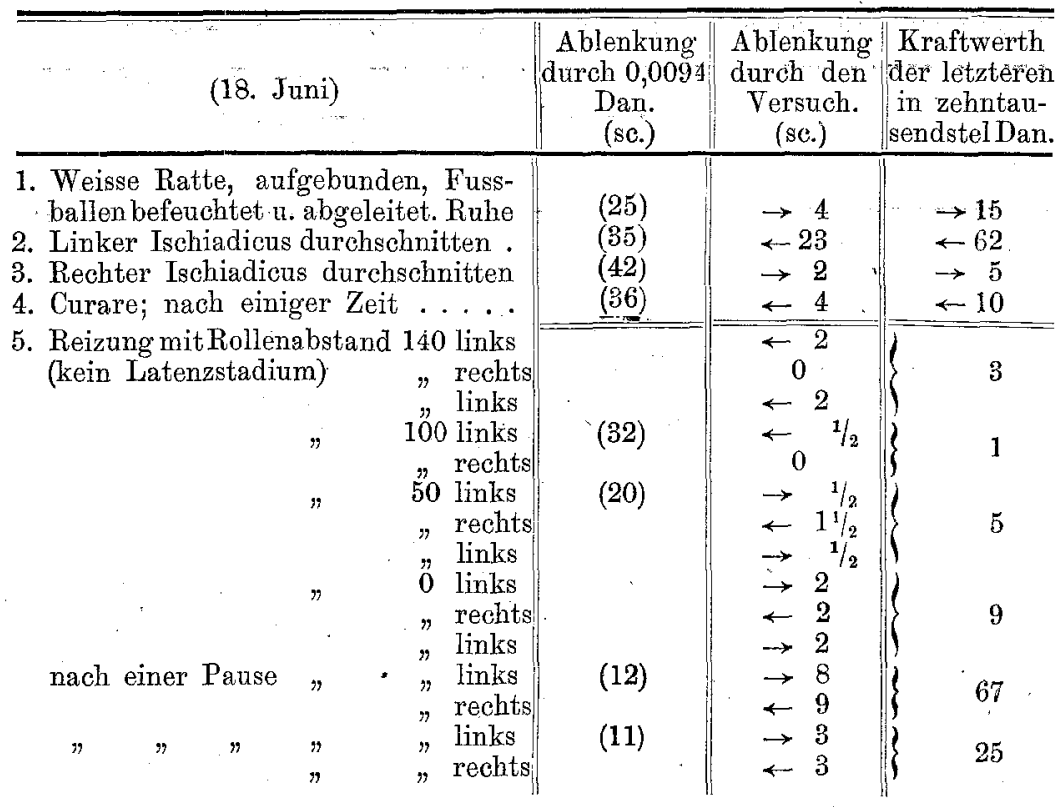

Endlich sei erwähnt, dass wir anch die Frage, ob an grösseren Drüsen Secretionsströme nachzuwèisen sind, bereits in Angriff genommen haben, freilich von vornherein in dem Bewusstsein, dass die Verhältnisse hier für die Ableitung von Strömen so ungiinstig wie möglich sind. In der That haben wir bisher nur negative Resultate zu verzeichnen. Die Versuche sind an der Submaxillardriise grosser Hunde angestellt. 'In beide Wharton'sche Gänge wurden dünnwandige möglichst weite Glascanuilen eingefiuhrt und so weit als möglich eingeschoben. Am äusseren Ende erweiterten sich beide Glasröhren zu einem flachen Trichterchen. Durch kurze Chordareizung wurden beide Röhren bis in den Trichter hinein mit Speichel gefiilt, und nun vom Inhalt beider Trichter symmetrisch abgeleitet und beide Chordae abwechselnd gereizt. Jedoch zeigte sich kein deutlicher Secretionsstrom.

Die Versuche ïber das Gebiet der Secretionsströme werden fortgesetzt werden.

\section{Zusatz von L. Hermann.}

Ich benutze diese Gelegenheit, um über meine in den Sommermonaten gesammelten Erfahrungen über die Secretionsströme der Haut kurz zu berichten. Man erinnert sich, dass ich die Ursache. 
der Abweichungen meiner Angaben von denen früherer Beobachter zum Theil in der Jahreszeit suchen zu können glaubte (vgl. dies Archiv XVII. p. 304). Indessen habe ich in zahlreichen Versuchen an frisch gefangenen Sommerfröschen (Esculenten) die gleichen Resultate erhalten wie an Winter- und Frühlingsfröschen. Nur ist mir im Sommer etwas häufiger als im Winter an der Rückenhaut ein aussteigender (negativer) Vorschlag zu dem einsteigenden (positiven) Hauptstrom begegnet, und in diesen Fällen war das Secret der Rückenhaut auch nicht rein alkalisch, sondern machte auf blauem Lacmuspapier gesprenkelte rothe Flecken, wie ich es schón an Winterfröschen zuweilen beobachtet hatte (vgl. meine frühere Arbeit p. 305). An der Unterschenkelhaut wurden durchaus keine anderen Resultate als im Winter und Frühling erhalten.

Zahlreiche Versuche, der schon früher erörterten, aber zweifelhaft gelassenen Ursache der beiden Secretionsströme näher auf die Spur zu kommen, sind bis jetzt erfolglos geblieben, so dass ich ibre Mittheilung voi der Hand unterlasse. Nur das sei erwähnt, dass in den Fällen, wo die Riickenhant negative Vorschläge ergab, der Ruhestrom besonders stark entwickelt schien.

(Aus dem physiologischen Laboratorium in Zürich.)

\section{Notizen zur Physiologie des Glykogens.}

Von

\section{B. Tuchsinger.}

I. Zur Bedeutung des Muskelglykogens.

Nachdem zuerst Nas s ${ }^{1}$ ) einen Verbrauch von Muskelglykogen dúrch Arbeit und Starre nachgewiesen und Weiss ${ }^{2}$ ) diese Erfahrung mit der verbesserten Brücke'schen ${ }^{3}$ ) Methode vollauf

1) Nasse, dieses Archiv II. 97-121. 1869.

2) Weiss, Wiener acad. Sitzungsberichte. LXIV. Band. II. Abtheilung. 1871.

3) Brücke, Wiener acad. Sitzungsberichte. LXIII. Band. II. Abtheilung. 1870 . 